\title{
Duodenal perforation in an adult with uncorrected Tetralogy of Fallot
}

Jamil Ahmad, Hira Bakhtiar, Maryam Karim

\section{Submitted}

December 22, 2019

Accepted

January 18, 2020

\section{Author Information}

From: Department of General Surgery, Rehman Medical Institute, Peshawar, Khyber Pakhtunkhwa, Pakistan

Professor Dr. Jamil Ahmad Consultant General \& Laparoscopic Surgery and Head of Department (Corresponding Author) Email:

jamil.ahmad@rmi.edu.pk

Dr. Hira Bakhtiar

Dr. Maryam Karim

House Officers

Citation: Ahmad J, Bakhtiar H, Karim M. Duodenal perforation in an adult with uncorrected Tetralogy of Fallot. [Case Report]. J Rehman Med Inst. 2020 JanMar;6(1):23-5.

\section{ABSTRACT} vomiting and jaundice. the work.

\section{INTRODUCTION} Gastrointestinal Stromal Tumor.
Intestinal perforation is a common cause of acute abdomen presenting in the Accident \& Emergency department. A rare case is presented of a 34-year-old male, known case of uncorrected Tetralogy of Fallot (TOF), developing sudden abdominal pain, bilious

The authors declared no conflict of interest. All authors contributed substantially to the planning of research, data collection, data analysis, and write-up of the article, and agreed to be accountable for all aspects of

Acute abdomen is a common presentation in the Accident \& Emergency department where intestinal perforation is one of the chief differential diagnoses. Perforation usually results from an underlying Peptic Ulcer Disease (PUD), mostly associated with use of Non-Steroidal AntiInflammatory Drugs (NSAIDs) and Helicobacter pylori (H. Pylori) infection. Other factors include ischemic perforation due to increased viscosity of blood, Zollinger-Ellison syndrome or

In routine practice, any patient presenting with abdominal pain, bilious vomiting and jaundice is first assessed for the possibility of acute cholecystitis; other conditions like intestinal perforation are seldom considered. With advances in radiological services, reaching a quick and accurate diagnosis has become achievable. Such a perforation is commonly attributed to PUD which results from an imbalance between stomach acidpepsin and mucosal defense barriers. ${ }^{1}$ Only $2-14 \%$ of patients with PUD present with acute abdomen due to perforation while $10-20 \%$ of patients have complications that can be treated conservatively. ${ }^{2}$ The incidence of these ulcers in Pakistan and India are 5:1 and 32:1 respectively. ${ }^{3}$ Timely diagnosis and identification of these perforations is of utmost importance in these cases and emergency surgical intervention is the preferred modality of treatment. ${ }^{4,5}$ Only sealed or controlled perforations can be managed conservatively or by interventional radiology. ${ }^{6}$

The presentation of intestinal perforation in an adult with uncorrected Tetralogy of Fallot is unheard of. Here we discuss a case of a 34-year- old male, known case of uncorrected Tetralogy of Fallot (TOF), presenting with abdominal pain, bilious vomiting and jaundice.

\section{CASE REPORT}

A 34-year-old male, known case of Tetralogy of Fallot, presented to Accident \& Emergency unit on the July 22, 2019 with complaints of abdominal pain and bilious vomiting for the past 2 days. He gave a history of being treated at the local hospital with analgesics and antibiotics, but his condition remained unchanged. On initial examination, his Blood Pressure was 103/68mmHg, Pulse 58/min, Temperature $98^{\circ} \mathrm{F}$, and a Glasgow Coma Scale (GCS) of 15/15. Grade 4 clubbing and jaundice was noticed. Systemic Examination revealed bilateral decreased breath sounds, a grade 4/6 pan systolic murmur and a tense, tender abdomen with audible bowel sounds. Resuscitative measures were taken, and relevant investigations performed, revealing Hemoglobin $20.5 \mathrm{gm} / \mathrm{dl}$, White Cell Count $14.67 \times 10^{9} / 1$, Neutrophils $87.1 \%$, Platelets 119 x 109/1, Total Bilirubin $7.53 \mathrm{mg} / \mathrm{dl}$, Alanine Transaminase (ALT) $10 \quad \mathrm{U} / \mathrm{L}, \quad$ Alkaline Phosphatase (ALP) $56 \mathrm{U} / \mathrm{L}$, Albumin $3.13 \mathrm{gm} / \mathrm{dl}$, and C-Reactive protein $50.73 \mathrm{mg} / \mathrm{dl}$. Serum electrolytes and Renal Function Tests were normal. Electrocardiogram (ECG) showed Sinus Rhythm with right axis deviation. Echocardiography revealed an ejection fraction of $65 \%$, cardiac situs solitus, levocardia, dilated right ventricle $(\mathrm{RV})$ with visually preserved function, large ventricular septal defect (VSD) with 50\% over riding of aorta, thickened pulmonic valve with pressure gradient of $87 \mathrm{mmHg}$, confluent pulmonary arteries, normal left aortic arch, dilated inferior vena cava and hepatic veins with no clot or pericardial effusion.

A provisional diagnosis of acute cholecystitis was made and an urgent CT- abdomen was performed which showed mild pneumoperitoneum and ascites with the possibility of underlying bowel perforation, likely in proximal duodenum along with cholelithiasis and bilateral small pleural effusions as seen in Figure 1 (a-d). Cardiology and Anesthesia departments were consulted, patient was declared vitally stable and fit for surgery under high-risk category, ASA-4 (the American Society of Anesthesiology score). 
Patient was shifted to the operation theater (OT) for an urgent Exploratory Laparotomy under General Anesthesia (GA) with
High-Risk Consent.

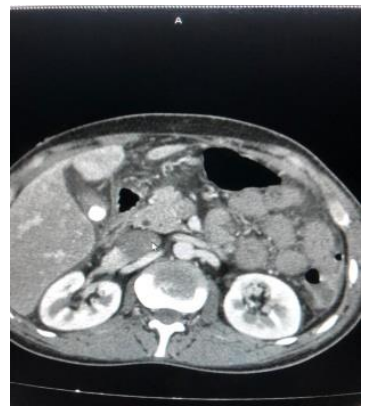

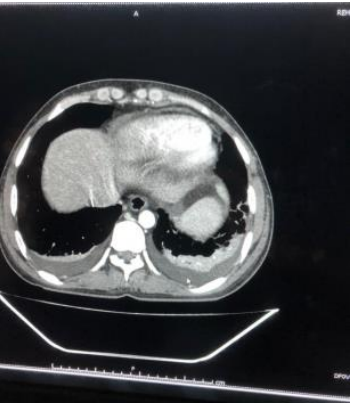
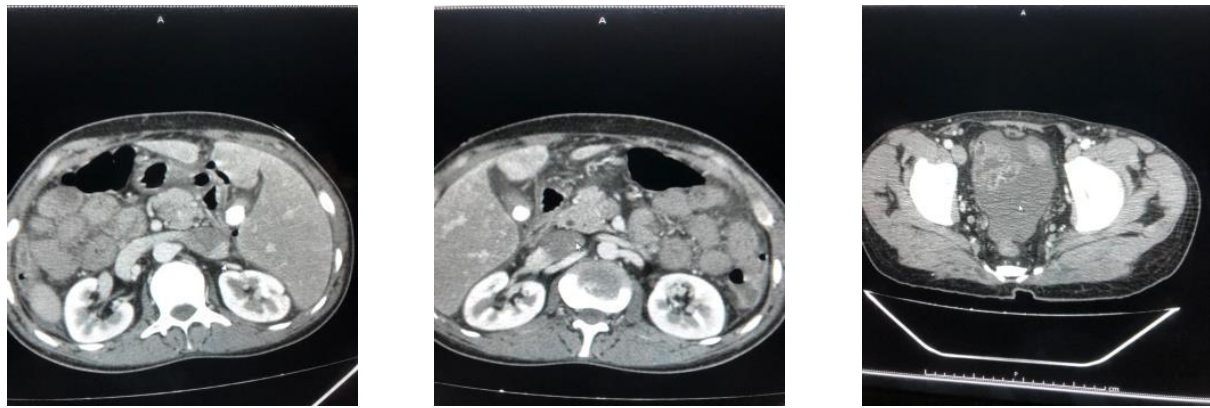

Figure 1 (a-d): CT scans of abdomen and pelvis showing mild pneumoperitoneum with ascites, possibility of duodenal perforation, and cholecystitis; bilateral small pleural effusions are seen.

In the Operation Theater, a Central Venous Pressure (CVP) line was secured after attempts to pass Peripheral Venous Line failed due to hyper viscosity of blood. Along with this, a right radial arterial line was also secured for monitoring of blood pressure. Patient was administered Inhalational Anesthesia alongside ultrasound guided bilateral rectus sheath blocks. An upper midline incision was given; intra-abdominal findings included an anterior perforation about $1 \mathrm{~cm}$ in first part of duodenum and a single large gallbladder stone. Cholecystectomy was performed. Following cholecystectomy, the omentum was mobilized showing blue-black colored vessels, the perforation site was identified and sealed with Graham patch. The peritoneal cavity was washed 4 times with Normal Saline and a drain placed in subhepatic space. Abdomen was closed with polydioxanone (PDS) suture. Since patient was High Risk for Deep Vein Thrombosis, his position was changed twice during the procedure. Entire Surgery was accomplished in 60 minutes.

After recovery, he was kept Nil by Mouth in Surgical Intensive Care Unit (SICU) on Ringer Lactate $100 \mathrm{ml} / \mathrm{hour}$. Limb physiotherapy was performed regularly, Elastic stockings were used and pressure dressing applied due to slight bleeding from surgical site. Overall, the ICU stay was uneventful, and the patient was shifted back to ward on second post-op day.

Patient was allowed oral intake of fluids once shifted to Ward along with Intravenous (I/V) painkillers and antacids. Stool for H. pylori was also sent on second post-op day, but result showed negative findings. Cardiothoracic surgery was consulted regarding the possibility of correcting TOF in this patient. Patient and attendants were counseled and advised surgery after 2 months. Patient was discharged on $4^{\text {th }}$ postoperative day in stable condition and advised follow up in 1 week. Consent for writing a case report was duly taken from the patient on the day of discharge.

\section{DISCUSSION}

Uncorrected Tetralogy of Fallot is an uncommon entity in itself with $70 \%$ affected people dying within the first decade of life. ${ }^{7}$ Amongst these, the incidence and likely causes of gastrointestinal perforation in adults with TOF cannot be accurately assessed due to unavailability of data. Although one study highlighted the possibility of mesenteric ischemia due to polycythemia ${ }^{8}$ while another discussed the likelihood of false negative $H$. pylori testing that is commonly encountered, particularly with tests that depend upon the number of organisms (including the stool antigen test) when the patient is taking concurrent antibiotics, proton pump inhibitors, or bismuth. $^{9}$

Generally PUD is considered the most prevalent cause of ulceration and perforations encountered in recent times. ${ }^{10}$ They are encountered either in the duodenum or the stomach, duodenal ulcers being more prevalent than gastric ulcers, where if perforation was to occur, the likely site involved would be the anterior wall of the duodenum $(60 \%)$, antral region $(20 \%)$ or lesser-curvature gastric ulcers $(20 \%) .{ }^{11}$

The principal factors involved in development of PUD are NSAIDs and $H$. pylori infections ${ }^{12}$ but in this case $H$. pylori test came negative whereas the patient reported no history of NSAID use. Other less common factors include steroid use, smoking, neoplastic disorders and trauma; ${ }^{7}$ idiopathic perforations constitute around $12.9 \% .^{2}$ Ultimately there are four mechanisms that can lead to a perforation of the intestinal tract: Ischemia, Infection, Erosion, and Physical disruption. ${ }^{6}$

Surgical management of these perforations remains the mainstay of treatment. Nonsurgical treatment is only advised where there is a proven sealing of the perforation site. ${ }^{11}$ Disadvantages of conservative management include a higher mortality rate in case it fails and lack of the benefit of surgery as a diagnostic tool in case the patient had been misdiagnosed. ${ }^{6,11}$

The gold standard method of surgical closure of the perforation is the so-called Graham patch. ${ }^{1}$ The omental graft used in this procedure provides the stimulus for fibrin formation and therefore quicker healing. ${ }^{11}$ Another cornerstone for such surgery is irrigation of peritoneal cavity. Peritoneal toilette with 6-10 liters and even up to 30 liters of warm saline are recommended. ${ }^{11,13}$ In modern day medicine, laparoscopic surgery is gaining popularity, but suturing techniques with this method take much longer time and it can be tough on inflamed tissues surrounding a perforation due to their friability. ${ }^{14}$

In a patient with TOF, any non-cardiac surgery becomes all the more difficult due to anesthetic considerations as well as increased chances of postoperative complications. Such patients require close observation in a high-dependency unit or intensive care unit to increase chances of catching any arrhythmia, 
cardiac ischemia, dehydration, pain, respiratory issues and other complications before they cause deleterious effects. ${ }^{15}$

Regarding the cause of this perforation, the patient had no relevant history. Literature reports that many times the etiology is unclear, and a perforation is spontaneous. Currently available data lack information regarding patients of TOF presenting with perforation of the bowel. In any case, an urgent exploratory laparotomy is essential in these patients to reduce the morbidity and mortality.

Often the patient is unwilling to investigate further, or false negative results are reported due to the ongoing treatment. Also, such emergencies in patients with other co-morbidities can be difficult to manage especially when surgery is the primary modality of treatment.

\section{CONCLUSION}

Even though the most common cause of a duodenal perforation is deemed to be the presence of underlying Peptic Ulcer Disease, the possibility of ischemic changes due to polycythemia or idiopathic causes cannot be ruled out. The swift and cautious response in high-risk individuals is more likely the cornerstone of successfully navigating through such unusual presentations.

\section{REFERENCES}

1. Chung KT, Shelat VG. Perforated peptic ulcer-an update. World J Gastrointest Surg. 2017 Jan 27;9(1):1-12.

2. Di Saverio S, Bassi M, Smerieri N, Masetti M, Ferrara F, Fabbri C, et al. Diagnosis and treatment of perforated or bleeding peptic ulcers: 2013 WSES position paper. World J Emerg Surg. 2014 Dec 1;9(1):45

3. Lakhani S, Bushra R, Alam S, Shafiq Y. Evaluation of prescribing trends and cost analysis of peptic ulcer disease regimen in tertiary care setting. Pak J Biochem Mol Biol. 2015;48(3):69-73.

4. Arora BK, Arora R, Arora A. Modified Graham's repair for peptic ulcer perforation: reassessment study. Int Surg J. 2017 Apr 22;4(5):1667-71.

5. Søreide K, Thorsen K, Søreide JA. Strategies to improve the outcome of emergency surgery for perforated peptic ulcer. Br J Surg. 2014 Jan;101(1):e51-64.
6. Hafner J, Tuma F, Marar O. Intestinal Perforation. InStatPearls [Internet] 2019 Jun 28. StatPearls Publishing.

7. Parker JA, Grange C. Anaesthetic management of a parturient with uncorrected tetralogy of Fallot undergoing caesarean section. Int $\mathrm{J}$ Obstet Anesth. 2015;24(1):88-90.

8. Hussain A, Ansari T, Mahmood H, Ellul J. Recurrent small bowel infarction in a young man: polycythaemia or vasculitis? BMJ Case Rep. 2009;2009:bcr11.2008.1296.

9. Gisbert JP, Calvet X. Helicobacter pylorinegative duodenal ulcer disease. Alimentary pharmacology \& therapeutics. 2009 Oct;30(8):791-815.

10. Sirinthornpunya S. Prevalence of Helicobacter pylori infection in patients with peptic disease. J Med Assoc Thai. 2012 Mar 1;95(Suppl 3):S22-7.
11. Bertleff MJ, Lange JF. Perforated peptic ulcer disease: a review of history and treatment. Dig Surg. 2010;27(3):161-9.

12. Ramakrishnan K, Salinas RC. Peptic ulcer disease. Am Fam Physician. 2007 Oct 1;76(7):1005-12.

13. Lunevicius $\mathrm{R}$, Morkevicius $\mathrm{M}$ Management strategies, early results, benefits, and risk factors of laparoscopic repair of perforated peptic ulcer. World J Surg. 2005 Oct 1;29(10):1299-310.

14. Vasuki R, Arun PS, Moothedath RM. A rare case of spontaneous gastric perforation in an adolescent. Int Surg J. 2018 Aug 25;5(9):3171-4.

15. Menghraj SJ. Anaesthetic considerations in children with congenital heart disease undergoing non-cardiac surgery. Indian $\mathrm{J}$ Anaesth. $2012 \quad$ Sep;56(5):491-5. 